\title{
Fluorescence study on the interaction of human serum albumin with loureirin B
}

\author{
Xu Chen ${ }^{\mathrm{a}, *}$, Jia-Ming Ma ${ }^{\mathrm{a}}$, Ke-Lan Yong ${ }^{\mathrm{a}}$, Jing-Ci Lv ${ }^{\mathrm{b}}$ and Xia-Bing Zhang ${ }^{\mathrm{c}}$ \\ ${ }^{a}$ Experimental Center for Life Sciences, School of Life Sciences, Shanghai University, Shanghai, China \\ ${ }^{\mathrm{b}}$ College of Sciences, Shanghai University, Shanghai, China \\ ${ }^{\mathrm{c}}$ Department of Biomedical Engineering, University of Southern California, Los Angeles, USA
}

\begin{abstract}
The interaction between loureirin B (Lour B) and human serum albumin (HSA) was investigated by fluorescence and UV-vis absorption spectroscopy. Experimental results indicated that loureirin B had a strong ability to quench the intrinsic fluorescence of HSA through a dynamic quenching procedure. The fluorescence quenching data revealed that the quenching constants $\left(\mathrm{K}_{\mathrm{SV}}\right) 2.68 \times 10^{4}, 3.30 \times 10^{4}$ and $4.10 \times 10^{4} \mathrm{l} / \mathrm{mol}$ at 300,310 and $320 \mathrm{~K}$, respectively. Based on the thermodynamic parameters obtained, the positive values of enthalpy change $\Delta H$ and entropy change $\Delta S$ suggested that hydrophobic forces played a major role in the interaction of Lour B with HSA. According to Förster theory of energy transfer, the distance $r$ between HSA and Lour B was calculated to be $2.85 \mathrm{~nm}$. Furthermore, the effect of Lour B on the conformation of HSA was analyzed by synchronous fluorescence and three-dimensional fluorescence spectra.
\end{abstract}

Keywords: Fluorescence spectra, UV-vis spectra, loureirin B, human serum albumin, thermodynamic parameters

\section{Introduction}

Dragon's blood is one of the renowned traditional medicines, which from Dranaena cochinchinensis (Lour). S.C. Chen is named 'Resina Draaconis' in China. Loureirin B (4'-2-hydroxy-2,4,6-trimethoxy dihydrochalcone, structure shown in Fig. 1), one of the main constituents of Resina Draaconis, which belongs to flavonoids, has functions as: antithrombotic, anti-blood stasis, anticoagulant, analgesic, blood circulation promotion, reducing high level of blood sugar and other important therapeutic activities [5, $8,9,15,17,23]$.

Human serum albumin (HSA) is the most abundant protein in the circulatory system. The principle function of HSA is to transport many exogenous and endogenous molecules and metabolites, while it is also capable of binding with a large number of drugs [2,10,11]. Since drug-HSA interaction has a great significance in pharmacology, it would be helpful in providing a theoretical basis and experimental model for the screening and designing of new drugs in vitro.

The present work reported on the affinity of Lour B-HSA and thermodynamics was under physiological conditions. It was designed to examine the mechanism of fluorescence quenching, the quenching constants, the thermodynamic parameters, the major force type and the distance $r$ between HSA and

\footnotetext{
* Corresponding author: Xu Chen, Experimental Center for Life Sciences, School of Life Sciences, Shanghai University, 99 Shang Da Road, Shanghai, 200444, China. Tel.: +86 2166134592 205; Fax: +86 21 66134845; E-mails: xuchen@ staff.shu.edu.cn, yschenxu@ sina.com.
} 
<smiles>COc1cc(OC)c(CCC(=O)c2ccc(O)cc2)c(OC)c1</smiles>

Fig. 1. Chemical structure of loureirin B.

Lour B by fluorescence and UV-vis absorption spectroscopy. Additionally, the effect of Lour B on the HSA conformation was discussed by synchronous fluorescence and contour fluorescence spectra. The study on Lour B may afford valuable information for the clinical pharmacology and the mechanism of the medicine transfer in the body.

\section{Experimental section}

\subsection{Materials}

Loureirin B (National Institute for the Control of Pharmaceutical and Biological Products, China) was directly dissolved in anhydrous alcohol to prepare stock solution at final concentration of $1.22 \times$ $10^{-2} \mathrm{~mol} / \mathrm{l}$. The stock solution $(1.54 \mu \mathrm{mol} / \mathrm{l})$ of human serum albumin (approximately $99 \%$ and fatty acid free; Sigma Chemical Company, USA), was prepared in $0.1 \mathrm{~mol} / \mathrm{l}$ Tris- $\mathrm{HCl}$ buffer with $\mathrm{pH}=7.4$ containing $0.1 \mathrm{~mol} / \mathrm{l} \mathrm{NaCl}$. Double distilled water was used throughout.

\subsection{Apparatus}

Fluorescence measurements were performed on a spectrofluorimeter Model LS-55 (PerkinElmer, USA), The absorption spectra were recorded on Cary100 UV-vis spectrophotometer (Varian, Australia). The $\mathrm{pH}$ values were measured on a pHS-3C Digital pH-meter (Shanghai Lei Ci device Works, China).

\subsection{Procedures}

\subsubsection{Lour B-HSA interactions}

$3.0 \mathrm{ml}$ solution in $1.0 \mathrm{~cm}$ quartz cells containing $1.54 \mu \mathrm{mol} / \mathrm{l} \mathrm{HSA}$ was titrated by successive additions of a $1.22 \times 10^{-2} \mathrm{~mol} / \mathrm{l}$ stock solution of Lour B, and the concentration of Lour B was varied from 0 to $40.67 \mu \mathrm{mol} / \mathrm{l}$. Fluorescence spectra were recorded at 300,310 and $320 \mathrm{~K}$ in the continuous range of $300-550 \mathrm{~nm}$ upon excitation at $280 \mathrm{~nm}$ in each case. Both excitation and emission bandwidths were set at $6 \mathrm{~nm}$. The synchronous fluorescence spectra and three-dimensional fluorescence spectra of HSA in the absence and presence of increasing amount of Lour B were also recorded.

\subsubsection{UV-vis absorption measurements}

The absorption spectra of HSA in presence and absence of Lour B were noted down in the continuous range of 240-350 nm. HSA concentration was fixed at $1.54 \mu \mathrm{mol} / \mathrm{l}$ while the concentration of Lour B was varied from 0 to $40.67 \mu \mathrm{mol} / \mathrm{l}$. 


\subsubsection{Energy transfer between HSA and Lour B}

The absorption spectrum of Lour B $(1.54 \mu \mathrm{mol} / \mathrm{l})$ and the emission spectrum of HSA $(1.54 \mu \mathrm{mol} / \mathrm{l})$ were recorded in the continuous range of $300-550 \mathrm{~nm}$. Then, the overlap of the UV-vis absorption spectrum of Lour B with the fluorescence emission spectrum of HSA was used to calculate the energy transfer.

\section{Results and discussions}

\subsection{Analysis of fluorescence quenching of HSA by Lour B}

The intrinsic fluorescence of HSA comes from the tryptophan, tyrosine and phenylalanine residues. The fluorescence intensity of the protein can be decreased by a variety of molecular interactions, such decrease in intensity is called the fluorescence quenching [12]. The fluorescence was mainly contributed by the Trp214 residue alone. Figure 2 showed the fluorescence emission spectra of HSA with various amount of Lour B upon excitation at $280 \mathrm{~nm}$. Under the same conditions, Lour B did not show any fluorescence intensity upon excitation at $280 \mathrm{~nm}$ (Fig. 7(b), Contour spectra of Lour B). So the contribution of the Lour B could be neglected when measuring the protein fluorescence emission spectra. The influence of dilution on HSA solutions ignored because the volume of Lour B was very small.

The effect of Lour B on HSA fluorescence intensity was shown in Fig. 2. It could be seen that the fluorescence intensity of HSA dropped regularly with the increase of Lour B concentration, along with a slight red shift. This observation implied that the microenvironment changes were induced by the interactions of Lour B-HSA.

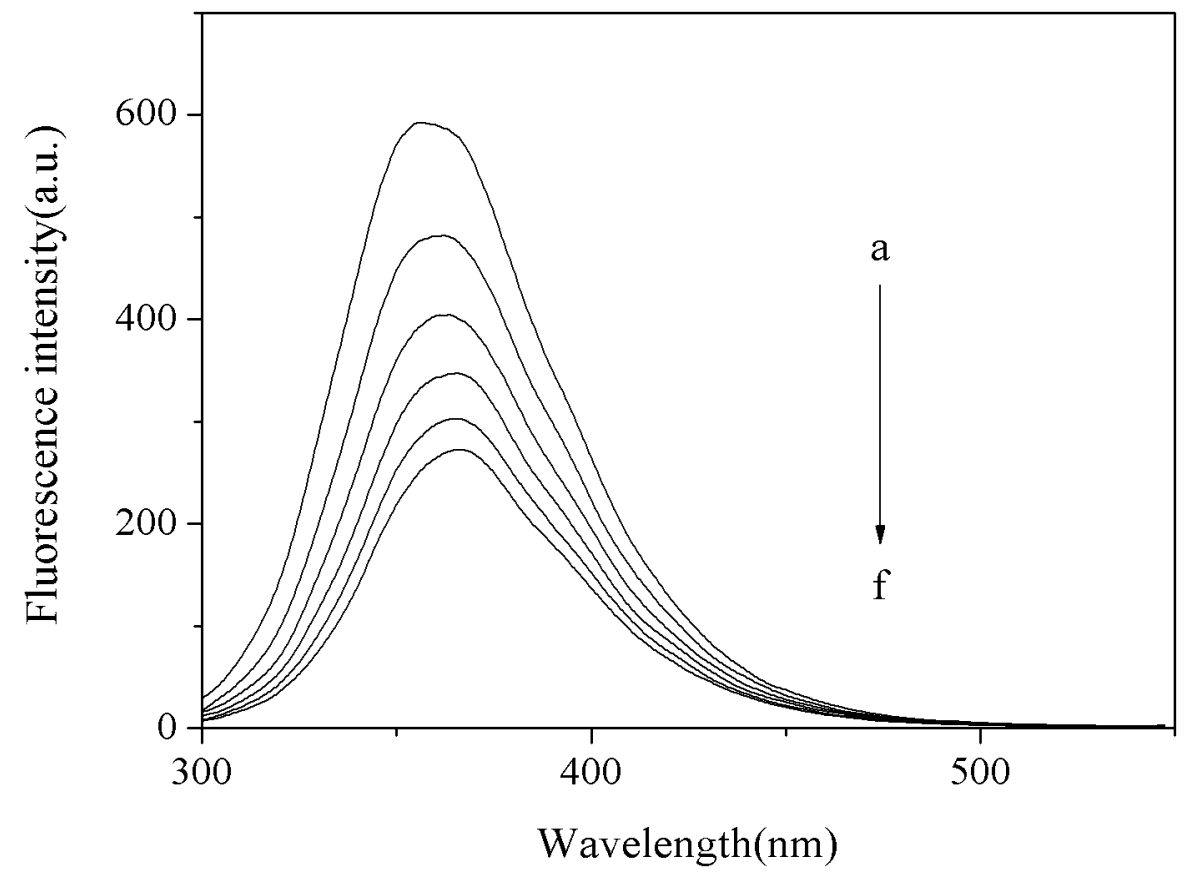

Fig. 2. Emission spectra of HSA in the presence of various loureirin B concentration. $C_{\mathrm{HSA}}=1.54 \mu \mathrm{mol} / \mathrm{l} ; C_{\mathrm{LourB}}: 0$ (a), 8.13 (b), 16.27 (c), 24.40 (d), 32.53 (e) and $40.67 \mu \mathrm{mol} / \mathrm{l}$ (f) $\left(\lambda_{\mathrm{ex}}=280 \mathrm{~nm}, T=310 \mathrm{~K}\right)$. 


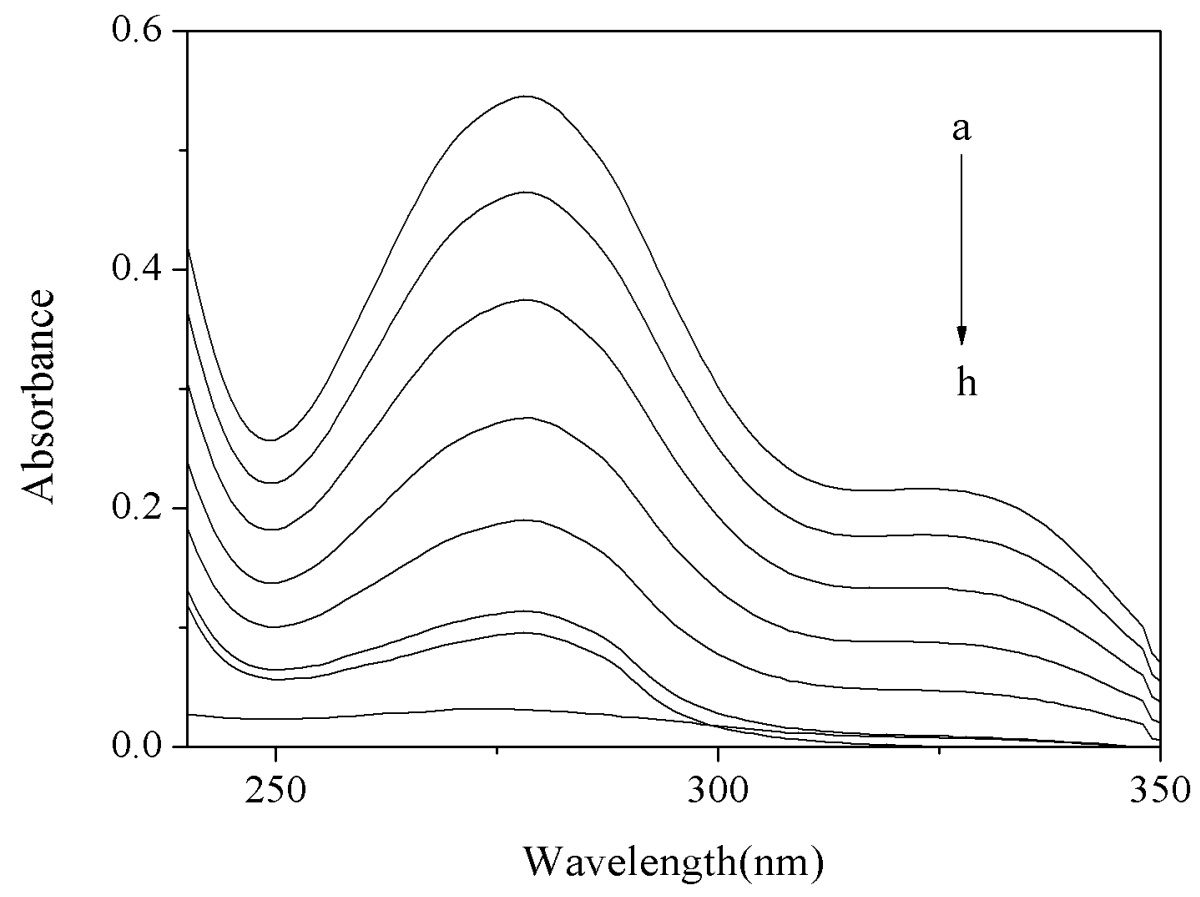

Fig. 3. UV-vis absorption spectra of HSA in the presence of different loureirin B concentration 40.67 (a), 32.53 (b), 24.40 (c), 16.27 (d), 8.13 (e), 1.54 (f) and $0 \mu \mathrm{mol} / \mathrm{l}(\mathrm{g})$; the spectra of loureirin B: $1.54 \mu \mathrm{mol} / \mathrm{l}(\mathrm{h})$.

\subsection{UV-vis spectra}

No complex formation between Lour B and HSA was evident from UV-vis absorption spectral data in Fig. 3. It was obvious that the maximum peak position of UV absorption of HSA did not change with the increase of Lour B concentration [4].

\subsection{Fluorescence quenching mechanism of HSA by Lour B}

Quenching can be induced by dynamic and static process [13]. The dynamic and static quenching can be distinguished by the results at different temperatures. As dynamic quenching only effect the fluorescent molecular excited states, it does not affect the absorption spectrometry [3]. Due to the conclusion of absorption spectrometry, it was supposed that the interaction of HSA with Lour B was dynamic quenching.

The fluorescence quenching data are usually analyzed by the Stern-Volmer equation [19]:

$$
\frac{F_{0}}{F}=1+\mathrm{K}_{\mathrm{Q}} \tau_{0}[Q]=1+\mathrm{K}_{\mathrm{SV}}[Q]
$$

where $F_{0}$ and $F$ are the fluorescence intensities of HSA in the absence and presence of Lour B, respectively; $\mathrm{K}_{\mathrm{Q}}$ is the quenching rate constant of the biomolecule, $\mathrm{K}_{\mathrm{SV}}$ is the Stern-Volmer dynamic quenching constant, $\tau_{0}$ is the average lifetime of the biomolecule without Lour $\mathrm{B}\left(\tau_{0}=10^{-8} \mathrm{~s}\right)$, and $[Q]$ is the concentration of Lour B. The values of $\mathrm{K}_{\mathrm{SV}}$ and other coefficient are listed in Table 1 . 
Table 1

Thermodynamic parameters of loureirin B-HSA interaction

\begin{tabular}{lcccccc}
\hline$T(\mathrm{~K})$ & $K_{\mathrm{SV}}\left(\times 10^{4} \mathrm{l} / \mathrm{mol}\right)$ & $R^{\mathrm{a}}$ & $\mathrm{SD}^{\mathrm{b}}$ & $\Delta H(\mathrm{~kJ} / \mathrm{mol})$ & $\Delta S(\mathrm{~J} / \mathrm{K} \cdot \mathrm{mol})$ & $\Delta G(\mathrm{~kJ} / \mathrm{mol})$ \\
\hline 300 & 2.68 & 0.99208 & 0.022 & & 138.73 & -25.43 \\
310 & 3.30 & 0.99941 & 0.029 & 16.19 & 138.74 & -26.82 \\
320 & 4.10 & 0.99569 & 0.054 & & 138.91 & -28.26 \\
\hline
\end{tabular}

Notes: ${ }^{\mathrm{a}} R$ is the correlation coefficient for the $K_{\mathrm{SV}}$ values; ${ }^{\mathrm{b}} \mathrm{SD}$ is the standard deviation for the $K_{\mathrm{SV}}$ values.

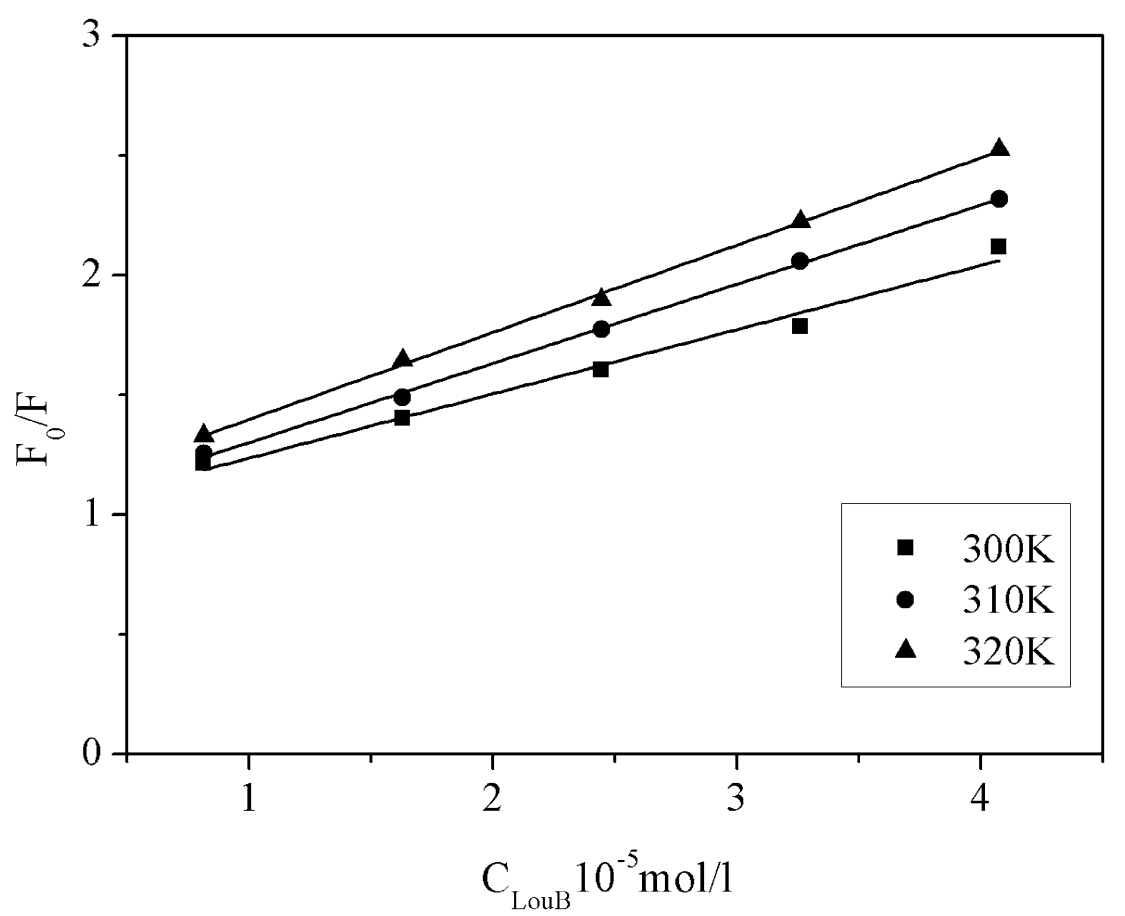

Fig. 4. Stern-Volmer curves of HSA fluorescence quenched by loureirin B.

It was concluded from Fig. 4 that the Stern-Volmer plots were linear and the quenching constants $K_{\mathrm{SV}}$ raised with increasing temperatures as shown in Table 1, these indicated that the fluorescence quenching process of HSA might be mainly governed by a dynamic collision quenching process.

\subsection{Thermodynamic parameters and nature of the binding forces}

The acting forces between a drug and a biomolecule may include hydrogen bonds, van der Waals forces, hydrophobic and electrostatic interactions, and so on [14]. Ross and Subramanian have characterized the sign and magnitude of the thermodynamic parameters associated with various individual kinds of interaction. For typical hydrophobic interactions, both $\Delta H$ and $\Delta S$ are positive, while negative for van der Waals forces and hydrogen-bond formation in low dielectric media. Moreover, the specific electrostatic interactions between ionic species in an aqueous solution is characterized by a positive $\Delta S$ value and a negative $\Delta H$ value [18]. Thermodynamic parameters were evaluated using the following 
equations:

$$
\begin{aligned}
& \Delta G=-R T \ln K, \\
& \ln \frac{K_{2}}{K_{1}}=\left[\frac{1}{T_{1}}-\frac{1}{T_{2}}\right] \frac{\Delta H}{R}, \\
& \Delta G=\Delta H-T \Delta S,
\end{aligned}
$$

where $\Delta G, \Delta H$ and $\Delta S$ are free energy change, enthalpy change and entropy change, respectively. $\Delta G$ reflects the possibility of reaction, $\Delta H$ and $\Delta S$ are the main evidence to determine acting forces [16]. In Eq. (3), $K$ is the quenching constant. The values of $\Delta H, \Delta S$ and $\Delta G$ at different temperatures were shown in Table 1. Because of $\Delta H>0$ and $\Delta S>0$, it was deduced that the main acting force between Lour B and HSA was mainly a hydrophobic interaction.

\subsection{Energy transfer from HSA to Lour B}

The fluorescence resonance energy transfer (FRET) can be divided into radiation energy transfer and non-radiation energy transfer, radiation energy transfer occurs when the fluorescence spectra of the donor is malformation. Figure 2 showed the fluorescence spectroscopy of the HSA was not distorted, Hence the molecular details of HSA-Lour B pair were supposed to be obtained from non-radiation energy transfer. According to Förster theory of non-radiation energy transfer [7,21], energy transfer is likely to happen if the following conditions are met: (1) the donor can produce fluorescence light; (2) fluorescence emission spectra of the donor and UV-vis absorption spectra of the acceptor have enough overlap; (3) the distance between the donor and the acceptor is less than $7 \mathrm{~nm}$. The energy transfer efficiency $E$, the distance $r$ and the critical energy transfer distance $R_{0}$, are given by the following equations:

$$
\begin{aligned}
& E=\frac{R_{0}^{6}}{\left(R_{0}^{6}+r^{6}\right)}, \\
& R_{0}^{6}=8.8 \times 10^{-25} K^{2} n^{-4} \Phi_{D} J, \\
& J=\frac{\sum F(\lambda) \varepsilon(\lambda) \lambda^{4} \Delta \lambda}{\sum F(\lambda) \Delta \lambda}, \\
& E=1-\frac{F}{F_{0}},
\end{aligned}
$$

where $R_{0}$ is the critical distance when the transfer efficiency equals to $50 \%, K^{2}$ is the spatial orientation factor of the dipole, $n$ is the refractive index of the medium, $\Phi_{D}$ is the fluorescence quantum yield of the donor and $J$ is the overlap integral between the donor emission spectrum and the acceptor absorption spectrum. $F(\lambda)$ is the fluorescence intensity of the fluorescent donor when the wavelength is $\lambda, \varepsilon(\lambda)$ is the molar absorption coefficient of the acceptor at the wavelength of $\lambda$. In the present case, $K^{2}=2 / 3$, $n=1.336$ and $\Phi_{D}=0.118[6]$.

The overlap of the fluorescence spectra of HSA and the absorption spectrum of loureirin B was shown in Fig. 5. According to Eqs (5)-(8), the calculated results showed that $J=3.561 \times 10^{-15} \mathrm{~cm}^{3} \cdot 1 / \mathrm{mol}$, $R_{0}=2.06 \mathrm{~nm}, E=0.13$ and $r=2.85 \mathrm{~nm}$. The distance $(r)$ was larger than $R_{0}$ but smaller than $7 \mathrm{~nm}$, which means most excited states elements was able to decay to the ground state. These data suggested 


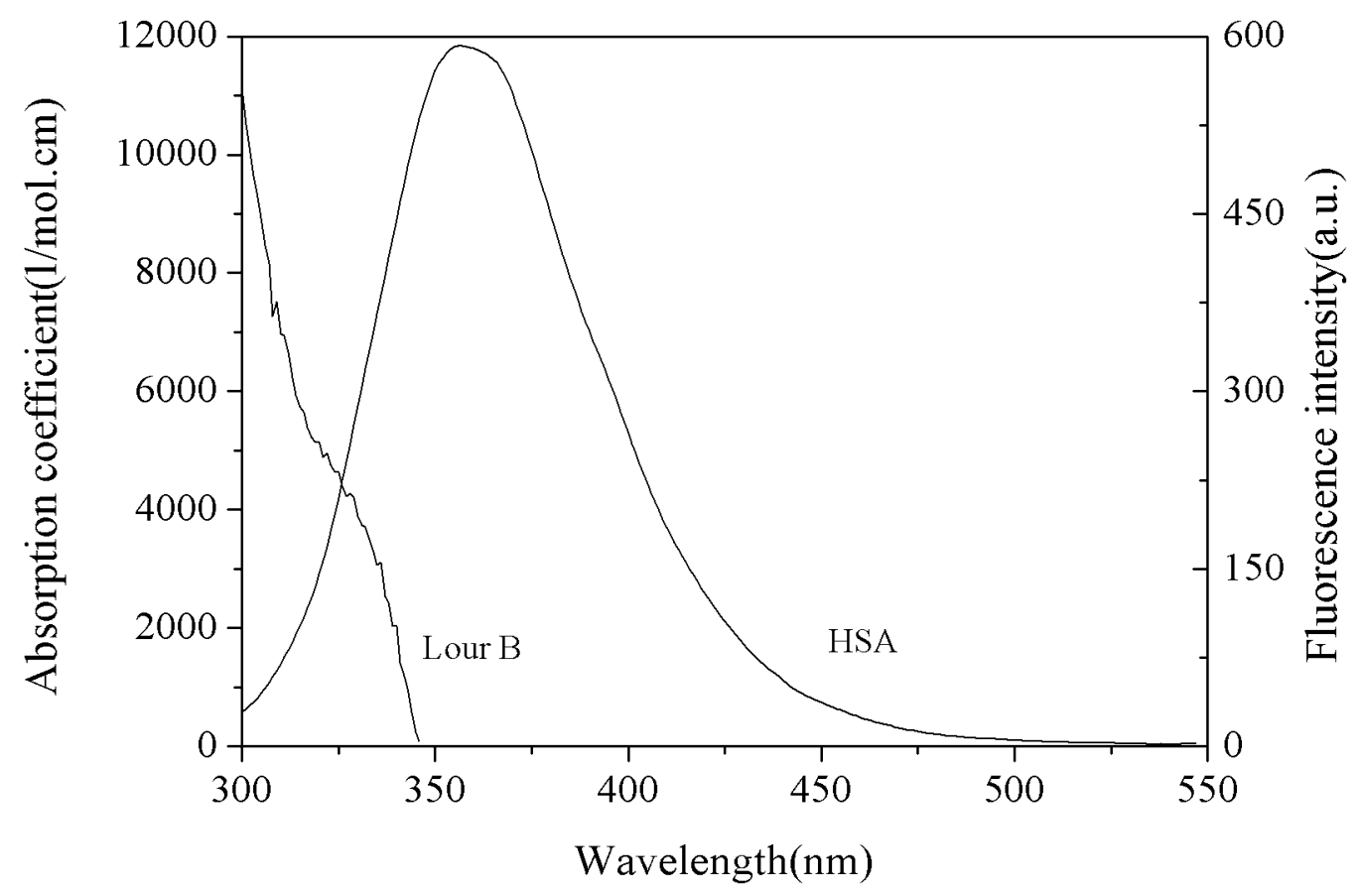

Fig. 5. Overlap of the fluorescence spectra of HSA and the absorption spectrum of loureirin B. $\mathrm{C}_{\mathrm{HSA}}=\mathrm{C}_{\mathrm{LourB}}=1.54 \mu \mathrm{mol} / \mathrm{l}$.

that the non-radiation energy transfer from HSA to Lour B could occur with high probability, but the dynamic quenching was played a major role in fluorescence quenching for Lour B-HSA.

\subsection{Effect of Lour B on the HSA conformation}

The synchronous fluorescence is a useful method to study the effect of drug on the protein conformation. The possible shift at the maximum emission wavelength of the amino acid residues is related to the changes of the polarity around the microenvironment. Therefore, the shift of the maximum emission wavelength reveals the alteration of molecule. The synchronous fluorescence can provide the characteristic information of the Try or Trp residues when the $\Delta \lambda$ value between excitation and emission wavelength is set at 15 or $60 \mathrm{~nm}[1,22]$.

The intrinsic fluorescence of HSA is mainly contributed by the Trp residue. The maximum emission wavelength of Trp residues had no shift with the addition of Lour B in Fig. 6(b). A slight red shift was observed from Fig. 6(a), which implied that the polarity around Tyr residues was increased and the hydrophobicity was decreased [20]. Therefore, the interaction of Lour B with HSA affect the conformation slightly, Fig. 6(a) showed the fluorescence intensity at $350 \mathrm{~nm}$ was increased with the addition of Lour B. It suggested that the intensity at $350 \mathrm{~nm}$ was induced by Lour B. The conclusion agreed with the result by the three-dimensional fluorescence spectra (Fig. 7b).

The contour spectra of HSA, Lour B and Lour B-HSA were shown in Fig. 7. It was concluded from Fig. 7(a) and (c) that the fluorescence intensity of HSA decreased obviously after the addition of Lour B and a slight red shift can be observed from the typical "fingerprint" of HSA. Thus, it revealed that the fluorescence intensity and the position of Lour B in Fig. 7(b) was the same as that in Fig. 7(c). We confirmed that the intrinsic fluorescence of the Lour B could be ignored while measuring the protein fluorescence emission spectra. The additional evidences came from the synchronous fluorescence and 

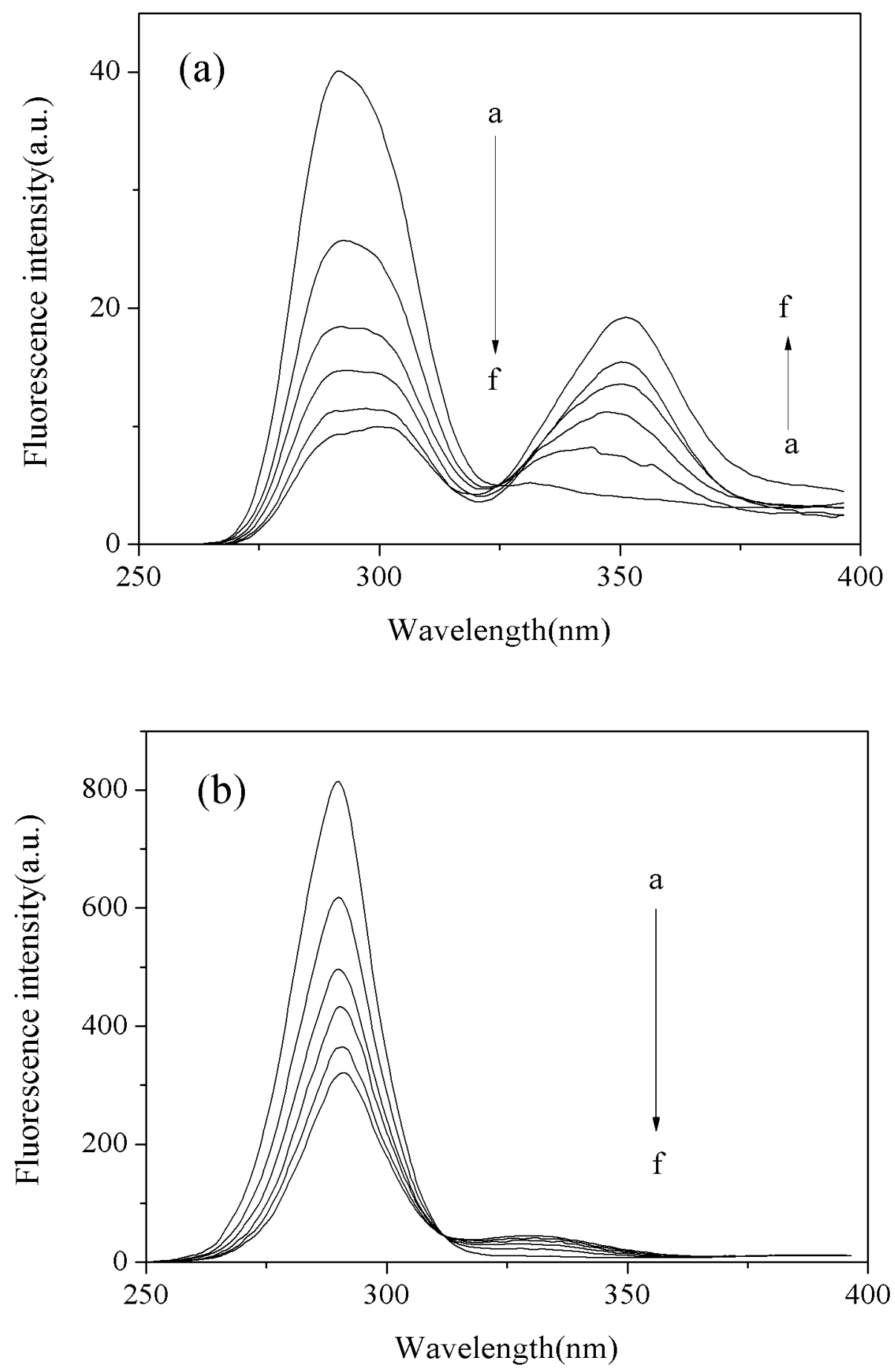

Fig. 6. Synchronous fluorescence spectra of HSA-loureirin B system. (a) $\Delta \lambda=15 \mathrm{~nm}$; (b) $\Delta \lambda=60 \mathrm{~nm}, \mathrm{C}_{\mathrm{HSA}}=1.54 \mu \mathrm{mol} / \mathrm{l}$; $\mathrm{C}_{\text {LourB }}$ : 0 (a), 8.13 (b), 16.27 (c), 24.40 (d), 32.53 (e) and $40.67 \mu \mathrm{mol} / \mathrm{l}$ (f) $(T=310 \mathrm{~K})$. 

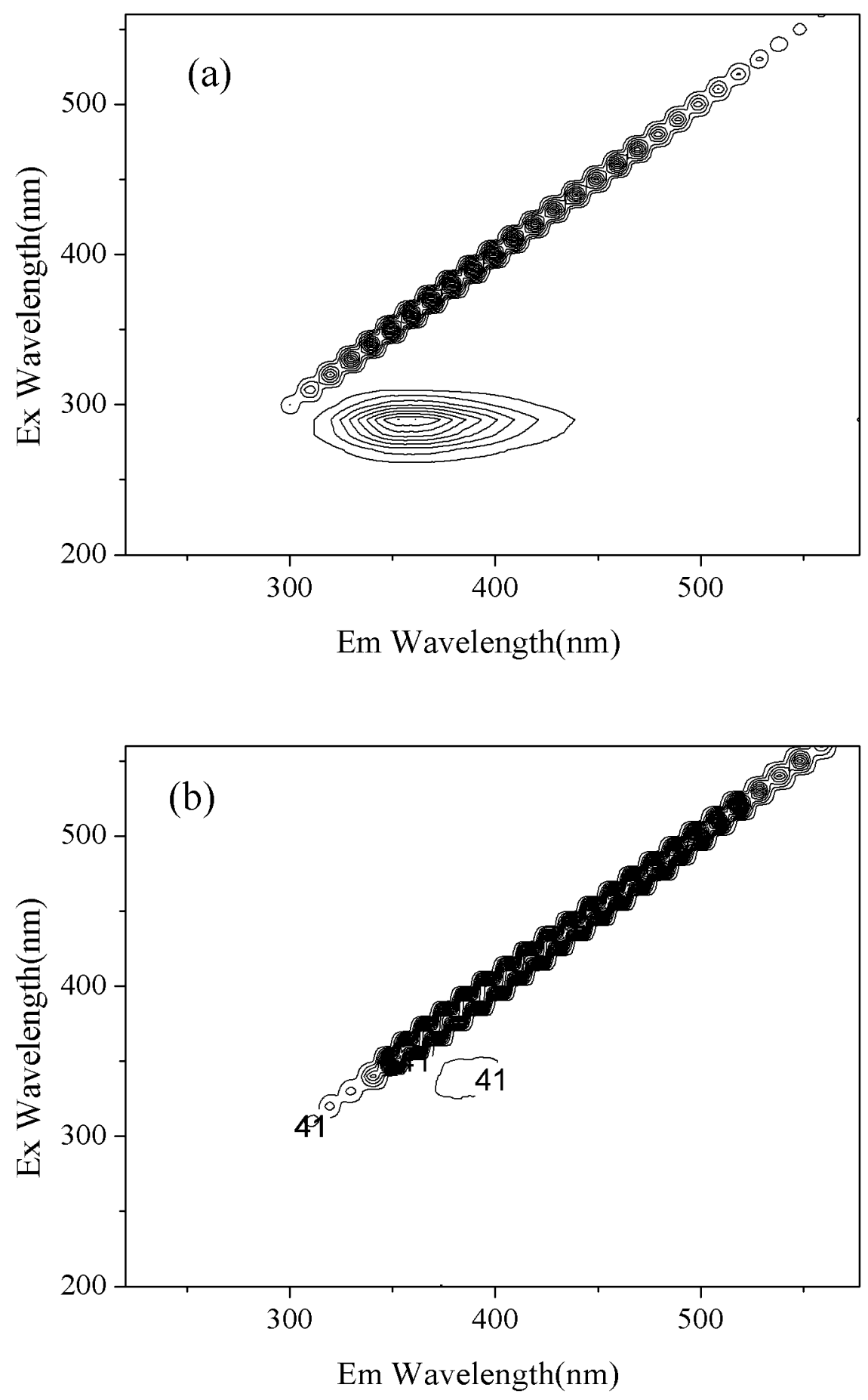

Fig. 7. Contour spectra of HSA (a), loureirin B (b) and loureirin B-HSA system (c). $\mathrm{C}_{\mathrm{HSA}}=1.54 \mu \mathrm{mol} / \mathrm{l}, \mathrm{C}_{\mathrm{LourB}}=$ $40.67 \mu \mathrm{mol} / \mathrm{l}$. 


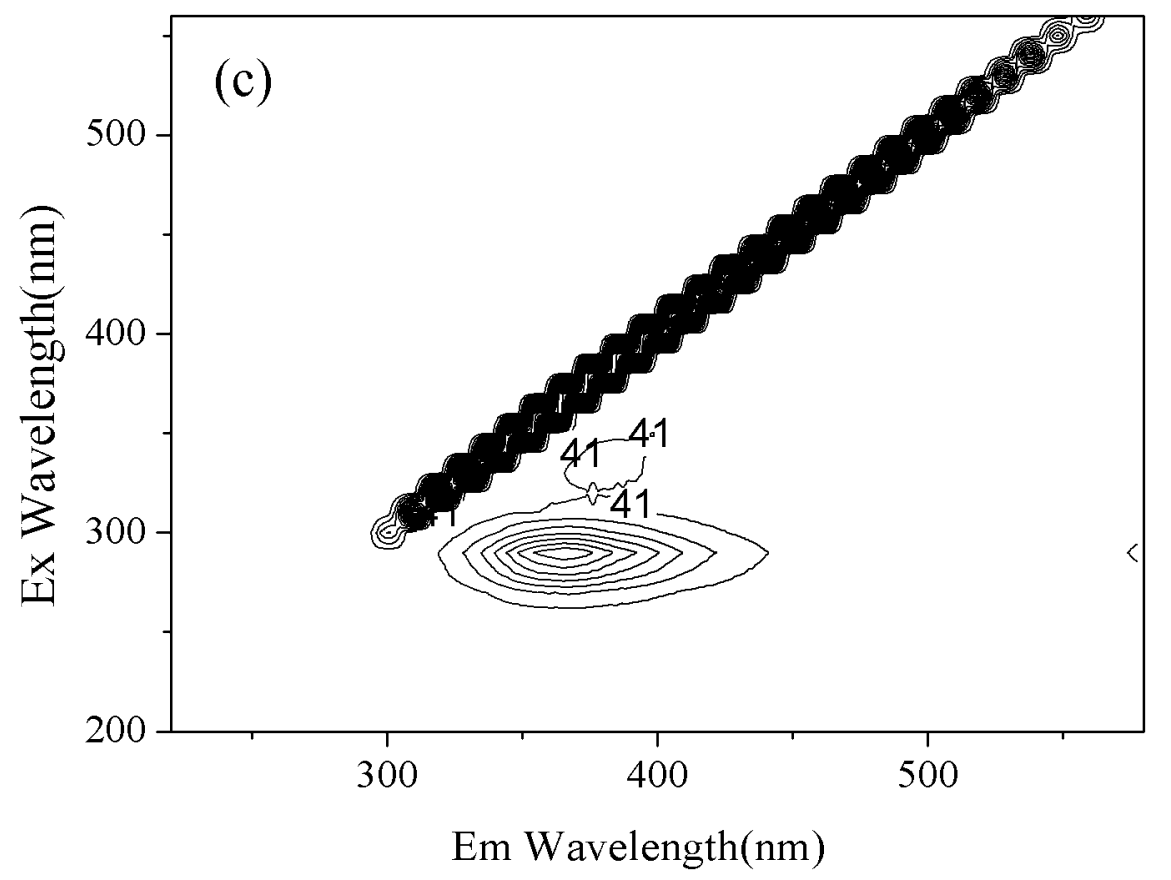

Fig. 7. (Continued).

three-dimensional fluorescence spectra, which indicated that the structure of HSA was slightly altered by Lour B binding.

\section{Conclusions}

Fluorescence spectroscopy was a powerful method to study molecular interactions involving proteins. The method can help us predict the structure of the chromophore molecule and the alteration of microenvironment. This paper provided an approach for studying the interaction of HSA with Lour B combined with fluorescence and UV-vis spectrophotometric techniques. Experimental results showed that Lour B had a strong ability to quench the intrinsic fluorescence of HSA through a dynamic quenching procedure. On the basis of the thermodynamic parameters, the binding reaction of Lour B with HSA was predominantly owing to hydrophobic interactions. From the fluorescence energy transfer studies, the critical transfer distance between tryptophan-214 in HSA and loureirin B was estimated to be $2.85 \mathrm{~nm}$. In addition, the results obtained from both synchronous fluorescence spectra and three-dimensional fluorescence spectra showed that the binding of Lour B with HSA can induce slight conformational changes in HSA. Therefore, this is also expected to open the door to the new avenues in screening and designing of drugs. This kind of study of interaction between HSA with loureirin B may be useful in pharmacology and clinical medicine.

\section{Acknowledgements}

This work was supported by Shanghai Science and Technology Development Funds (No. 08-JC1409-600), Science Foundation for the Excellent Youth Scholars of Shanghai University (No. 07-0112-07- 
002). The authors also express their deep thanks to Ming Cao and Shi-Jie Xu for help in fluorescence studies.

\section{References}

[1] W.C. Abert, W.M. Gregory and G.S. Allan, Anal. Biochem. 213 (1993), 407-413.

[2] D.C. Carter and J.X. Ho, Adv. Protein Chem. 45 (1994), 153-203.

[3] G.Z. Chen, X.Z. Huang, J.G. Xu, Z.Z. Zheng and Z.B. Wang, The Methods of Fluorescence Analysis, 2nd edn, Beijing Science Press, Beijing, 1990, pp. 112-145.

[4] F.L. Cui, L.X. Qin, G.S. Zhang, X.B. Liu, X.J. Yao and B.L. Lei, Bioorg. Med. Chem. 16 (2008), 7615-7621.

[5] J.G. Deng, H.B. Hung, Y.Q. Nong and Y. Liu, Guangxi J. Tradit. Chin. Med. 1 (2004), 44-45.

[6] D.E. Epps, T.J. Raub, V. Caiolfa, A. Chiari and M. Zamai, J. Pharm. Pharmacol. 51 (1999), 41-48.

[7] T. Förster and O. Sinanoglu (eds), Modern Quantum Chemistry, Vol. 3, Academic Press, New York, 1996, pp. 93-138.

[8] H.J. Gu, J.C. Lv, K.L. Yong, X. Chen, P.P. Liu and X.B. Zhang, J. Enzym. Inhib. Med. Ch. 24 (2009), 136-139.

[9] D. Gupta, B. Bleakley and R.K. Gupta, J. Ethnopharmacol. 115 (2008), 361-380.

[10] X.M. He and D.C. Carter, Nature 358 (1992), 209-215.

[11] J. Jacobsen and R. Brodersen, J. Biol. Chem. 258 (1983), 6319-6326.

[12] P.B. Kandagal, S. Ashoka, J. Seetharamappa, S.M.T. Shaikh, Y. Jadegoud and O.B. Ijare, J. Pharmaceut. Biomed. 41 (2006), 393-399.

[13] J.R. Lakowicz, Principles of Fluorescence Spectroscopy, 2nd edn, Kluwer Academic/Plenum Publishers, New York, 1999, pp. 238-249.

[14] D. Leckband, Annu. Rev. Bioph. Biom. 29 (2000), 1-26.

[15] H. Lu, J.B. Teng and H.E. Wu, J. Chin. Med. Mater. 26 (2003), 459-461 (in Chinese).

[16] A. Mallick, B. Haldar and N. Chattopadhyay, J. Phys. Chem. B 109 (2005), 14683-14690.

[17] D. Meksuriyen and G.A. Cordell, J. Nat. Prod. 51 (1988), 1129-1135.

[18] P.D. Ross and S. Subramanian, Biochemistry 20 (1981), 3096-3102.

[19] A. Sharma and S.G. Schulman, Introduction to Fluorescence Spectroscopy, Wiley, New York, 1999, pp. 58-59.

[20] K.H. Ulrich, Pharmacol. Rev. 33 (1981), 17-53.

[21] P. Yang and F. Gao, The Principle of Bioinorganic Chemistry, Beijing Science Press, Beijing, 2002, pp. 311-343.

[22] G.W. Zhang, Q.M. Que, J.H. Pan and J.B. Guo, J. Mol. Struct. 881 (2008), 132-138.

[23] Q.Y. Zhang, H. Zhu, H.Y. Cheng and Y.Q. Hu, Acta Acad. Med. CPAF 13 (2004), 69-71 (in Chinese). 


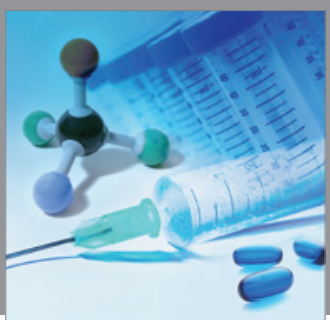

International Journal of

Medicinal Chemistry

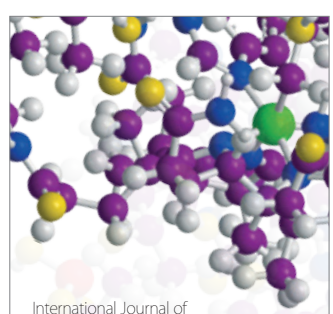

Carbohydrate Chemistry

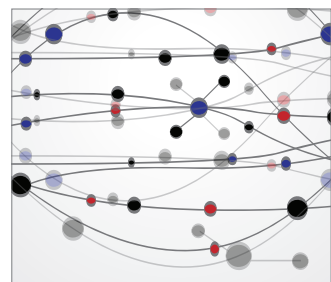

The Scientific World Journal
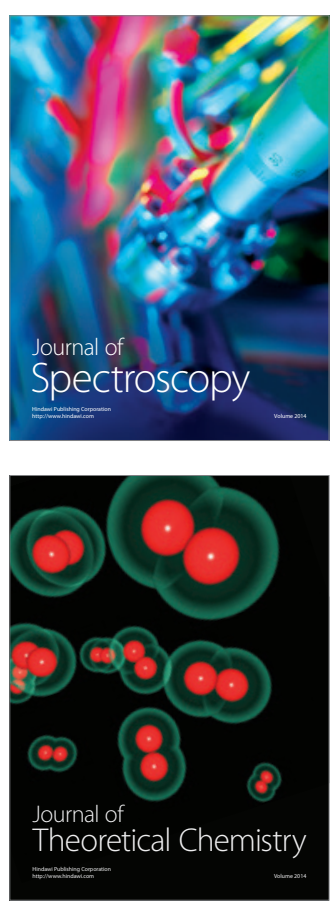
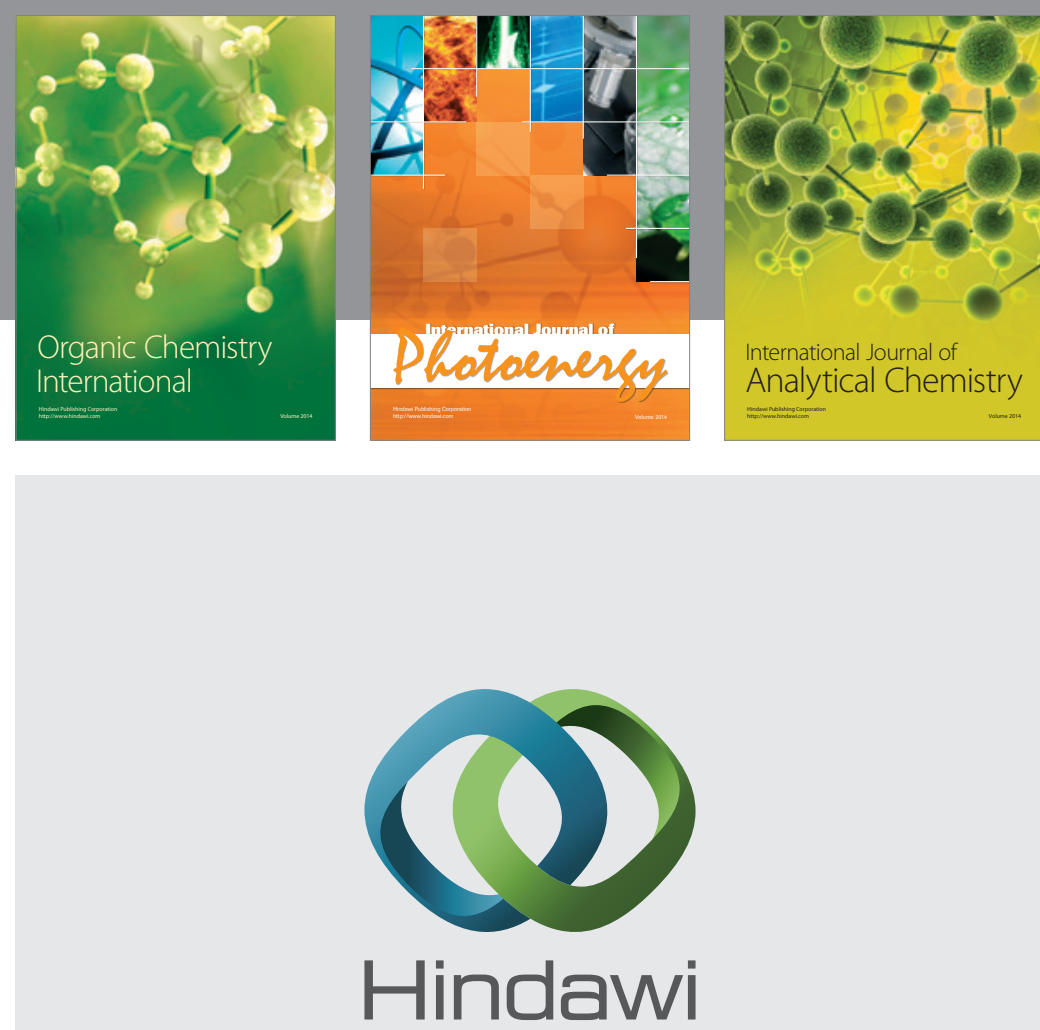

Submit your manuscripts at

http://www.hindawi.com
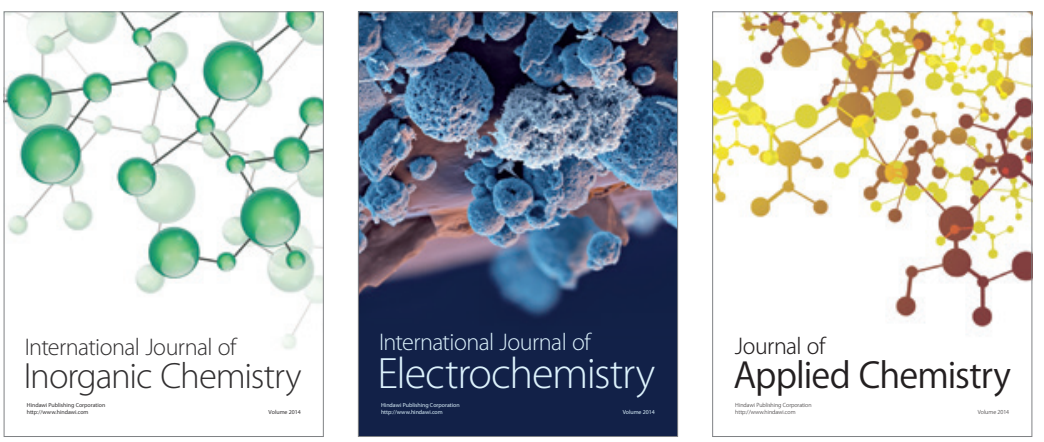

Journal of

Applied Chemistry
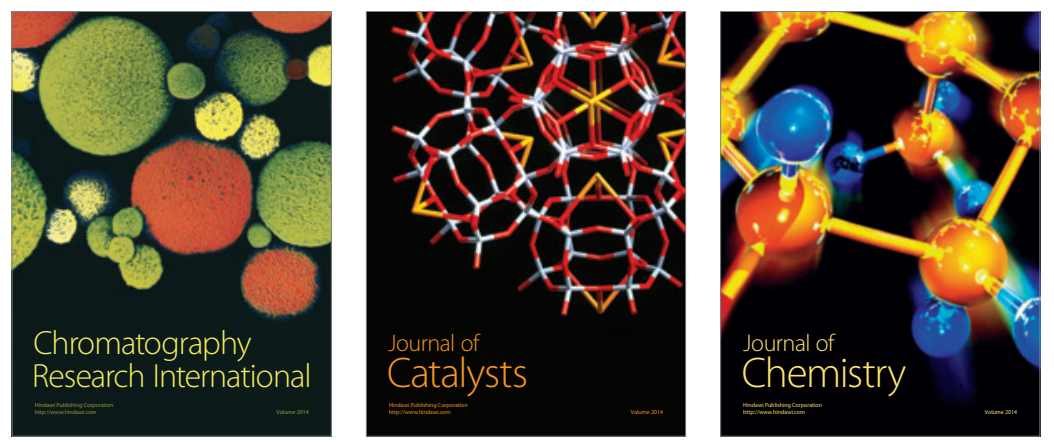
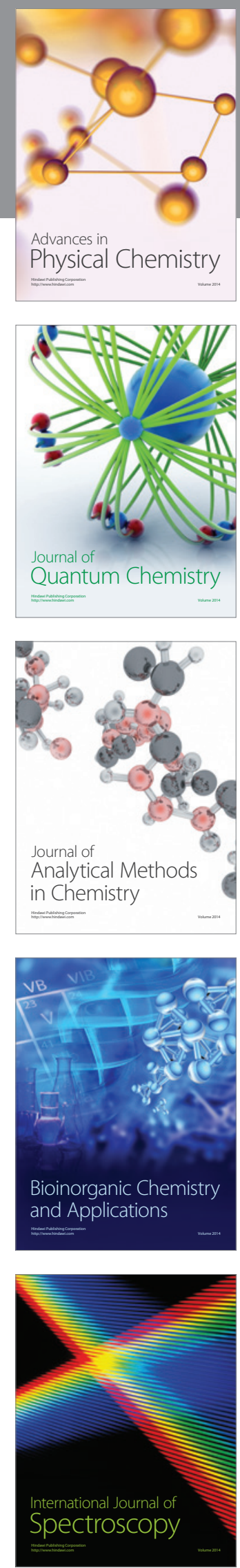